\title{
Theme issue on autism and technology
}

\author{
Gillian R. Hayes · Karrie G. Karahalios
}

Published online: 24 April 2011

(C) Springer-Verlag London Limited 2011

Autism spectrum disorder (ASD), also known as pervasive development disorder, includes impairments in social interaction, communication - both verbal and non-verbaland stereotypical or repeated behavior, interests, and activities [1]. A recent report by the Centers for Disease Control using data from the United States in 2006 indicated a rise in prevalence of ASD to 1 in 110 children: 1 in 70 boys and 1 in 315 girls [2]. Individuals across the spectrum can potentially benefit from the use of novel computing technologies, including assistive and augmentative technologies, social computational systems, sensing and context-aware systems, capture and access applications, wearable systems, and more.

The purpose of this theme issue is to bring together a set of research papers that (1) advance our understanding of technologies for autism, (2) discuss the role of technology in supporting individuals with ASD, their friends and families, and (3) provide examples of effective technologies for the diagnosis, management, and treatment of autism. Submissions to this theme issue came from an open call for papers. We selected seven papers to publish after multiple rounds of rigorous reviews. A large number of reviewers assisted us in the review process. To ensure high reviewing standards, three to four reviewers evaluated each

\footnotetext{
G. R. Hayes $(\bowtie)$

Department of Informatics, Donald Bren School

of Information and Computer Sciences, University of California,

Irvine, CA, USA

e-mail: gillianrh@ics.uci.edu

K. G. Karahalios

Department of Computer Science, University

of Illinois at Urbana-Champaign, Urbana, IL, USA
}

paper, and the guest editors provided additional feedback on all papers that are included in this issue.

The papers in this issue can be coarsely divided into three types. First, we include two papers focused on approaches to design and study technology for autism. Second are two papers that describe early design processes and methods and their resultant prototypes. Finally, there are three papers that cover deployments and evaluations of novel systems. These papers support a wide range of challenges for autism and impact a variety of stakeholders, age ranges, and diagnoses. Taken together, they provide a compelling overview of some of the latest work in this area, and we hope a call to action for other researchers to enter this important and rewarding space.

\section{Research approaches}

In the opening paper of this theme issue, "Developing Technology for Autism: an Interdisciplinary Approach," Porayska-Pomsta et al. argue for an interdisciplinary approach to designing interactive technologies for young children with ASD. They support this argument with data from the ECHOES project that focused on the development of a technology-enhanced learning environment. Likewise, the second article "Is Simplicity the Key to Engagement for Children on the Autism Spectrum" by Keay-Bright and Howarth presents technologies as simple and ambient. They lay out this argument through the use of case studies from a variety of experiences with children with ASD, in particular highlighting tangible interaction through an application called ReacTickles. These articles provide a strong basis for considering the remaining articles in this theme issue, all of which were developed using an interdisciplinary approach and many of which consider the 
trade-offs between various interaction modalities and the need for simplicity, flexibility, and complex functionality in varying forms.

\section{Novel interaction design for children with autism}

In the third and fourth papers in this theme issue, readers may explore the novel interaction approaches of tangible user interfaces and tablet applications. In "Using Tangible User Interfaces in Computer-Based Training Systems for Low-Functioning Autistic Children," Sitdhisanguan et al. describe the differences between tangible and traditional mouse-based interactions for computer-based learning and offer some design guidelines for successful tangible applications based on the results of their empirical studies of these interaction paradigms. In "Multitouch Tablet Applications and Activities to Enhance the Social Skills of Children with Autism Spectrum Disorders," Hourcade et al. similarly present design considerations for a variety of applications that make use of tablet platforms based on their iterative design process working with children with ASD, their teachers, and other stakeholders. The activities and design concepts presented in each of these papers can be well applied to other challenges in the autism and technology research space, particularly as access to tangible, tablet, and other novel form factors grows.

\section{Evaluations of autism technologies}

The final three papers of this theme issue demonstrate the challenges and opportunities of deploying research prototypes into homes, schools, and clinics. First, Westeyn et al. describe the design and development of sensor-enabled toys in "Monitoring Children's Developmental Progress Using Augmented Toys and Activity Recognition." Additionally, they report the results of an empirical study of children and adults using the toys in play sessions, highlighting both the technological aspects of these toys and their potential uses to support video annotation and other monitoring solutions. Second, Nazneen et al. describe the implementation of CRAFT, a research tool for allowing parents to selectively archive relevant data for monitoring their children in the natural environment at home in "Supporting Parents for In-home Capture of Problem Behaviors of Children with Developmental Disabilities." They further describe the results of an empirical study of parents using CRAFT in their homes. Finally, in "Embedded Capture \& Access: Encouraging Recording \& Reviewing of Data in the Caregiving Domain," Kientz describes an approach to encourage greater reviewing of recorded data, embedded access, for therapists working in homes and parents collecting developmental data. This paper highlights the empirical results from the deployment of multiple robust systems based on this concept of embedded access. Taken together, these three papers present both solid research prototypes and approaches for evaluating them through a variety of quantitative and qualitative methods.

\section{Conclusion}

The seven papers in this theme issue are by no means representative of all the work in autism and technology that might be of relevance to the readers of PUC. We sincerely hope readers who have become encouraged to get involved in this research space will continue to engage with the ubiquitous computing and human-computer interaction literature as well as consider attending the International Meeting for Autism Research, a multi-disciplinary conference that has for the last few years had an explicit technology track. The opportunities in this space are many and varied as this theme issue demonstrates. We encourage all readers to consider how their own work might be adapted to support the challenges of autism or how they might develop a new project or research trajectory in this fertile and significant research space.

Acknowledgments We thank Peter Thomas, Editor-in-Chief for his great support and effort throughout the publication process of this theme issue. We also thank the referees, many of whom do not traditionally review for PUC, for their important contributions to this process. Finally, we thank in particular the authors who submitted their work to this theme issue.

\section{References}

1. World Health Organization (2006) International statistical classification of diseases and related health problems, 10th edn. (ICD-10), F84, Pervasive developmental disorders

2. Rice C, Autism and Developmental Disabilities Monitoring Network Surveillance Year 2006 Principal Investigators (2006) Prevalence of autism spectrum disorders-autism and developmental disabilities monitoring network, United States. Surveill Summ 58(SS10):1-20 